IBIMA Publishing

Journal of Virology \& Microbiology

http://www.ibimapublishing.com/journals/JVM/jvm.html

Vol. 2013 (2013), Article ID 279685, 15 pages

DOI: $10.5171 / 2013.279685$

Research Article

\title{
Oxidative Stress Associated to Disease Progression and Toxicity during Antiretroviral Therapy in Human Immunodeficiency Virus Infection
}

\author{
Lizette Gil del Valle ${ }^{1}$, Rosario Gravier Hernández ${ }^{1}$ and Jorge Pérez Ávila ${ }^{2}$ \\ ${ }^{1}$ Clinical Pharmacology Department, Epidemiology Division, Institute of Tropical Medicine, Cuba \\ ${ }^{2}$ Institute of Tropical Medicine, Cuba
}

Received 26 November 2012; Accepted 6 December 2012; Published 16 January 2013

Academic Editor: Dariusz Pawlak

\begin{abstract}
Oxidative stress condition arises during imbalance between oxidants and antioxidants of diverse origin damaging both structure and function of tissues. Oxidative stress has been suggested as a cofactor during Human Immunodeficiency Virus Infection course involved in many aspects of disease pathogenesis as viral replication, inflammatory responses, decreased immune cell proliferation, loss of immune function, cellular apoptosis, chronic weight loss, and increased sensitivity to drug toxicity.

In this review, we provide an overview of the oxidative metabolism pathways involved in HIV infection, examine the potential impact of antirretroviral toxicity on oxidative damage and argue how the response contribute to co-morbidities. Moreover, we also discuss recent reports from observational and interventional studies which have led to an increasing interest in the possible benefits of micronutrient supplementation as a cost-effective strategy for improving oxidative and nutritional status in HIV infection. The general strategy and combination of these interventions represent an important complementary deal for HIV infection treatment in the era of high active antiretroviral therapy.
\end{abstract}

Keywords: HIV, oxidative stress, HAART.

\section{Introduction}

Free radical (FR)-rich primordial context, and the simultaneous development of antioxidants, were important for life improvement. Antioxidant adaptation involved subtle changes to structural integrity and compartmentalisation of reactants (e.g. keep reactive oxygen species (ROS) away from iron) within cells. ROS and antioxidants are essential parts of the basic biology and physiology mentioned by Halliwell (2006). ROS are meaningful also in neutralizing the ever-changing virulence of micro-organisms. The ability of immune system to sterilise a site of infection, by rapid production of ROS (superoxide anions $\mathrm{O}_{2} \cdot-$, hydrogen peroxide $\mathrm{H}_{2} \mathrm{O}_{2}$, hydroxyl radicals $\cdot \mathrm{OH}$, hypochlorous acid $\mathrm{HOCl}$, peroxynitrite $\mathrm{ONOO}^{-}$, etc.) can keep organism alive. The subjects, who could mount a robust immune response with vigorous yet coordinated ROS production, would be selected for survival refereed by Roberts et al (2010) and Sahnoun et al (1997).

Human immunodeficiency virus 1 (HIV-1), identified in 1983, remains a global health threat responsible for a world-wide

Copyright (C) 2013 Lizette Gil del Valle, Rosario Gravier Hernández, Jorge Pérez Ávila. This is an open access article distributed under the Creative Commons Attribution License unported 3.0, which permits unrestricted use, distribution, and reproduction in any medium, provided that original work is properly cited. Contact author: Lizette Gil del Valle E-mail: lgil@ipk.sld.cu 
pandemic. WHO (2011) reports showed that HIV infections evolved to acquired immune deficiency syndrome (AIDS) accompanied by opportunistic infections, severe metabolic and immune dysfunctions. Articles from Sonnerborg et al (1988), Stephens (2004), Pasupthi et al (2009), Coaccioli, et al (2010), Awodele et al (2012) and our group Gil et al (2003) have shown that humans infected with HIV are under chronic immune activation characterized by increase generation of ROS and perturbations of the antioxidant defence system. Oxidative stress (OS) has been detected in many tissues of HIV infected individuals using different biochemical's markers and diverse biotechniques. In research studies by Sonnerborg et al (1988), Stephens (2004), Pasupthi et al (2009), Coaccioli, et al (2010), Awodele et al (2012), Redhage et al (2009), Stephensen, et al (2007) and Wanchu et al (2009) the OS characterization has been made in populations from several countries and different risk groups using case-controls, cross-sectional and interventions studies. These researches show imbalance in redox status during the infection course which could be related to oxidative molecular damage, viral replication, micronutrient deficiency and inflammatory chronic response, all of them implicated in cellular apoptosis and decreased immune proliferation. Those imbalance included changes in glutathione (GSH), thioredoxin (TRX), superoxide dismutasa (SOD), ascorbic acid, glutathione peroxidase (GPx), tocopherol (TOC) and selenium (Se). In addition, hydroperoxides (HPO) and malondyaldehyde (MDA) elevated levels were found in both paediatrics and adults patients.

Several advances have been made in AIDS management since the introduction of the highly active antiretroviral therapy (HAART) in 1996. AIDS pandemic has stabilized on a global scale in 2008 with an estimated of 33 million people infected worldwide reported by UNAIDS (2011). In more recent years, as the number of available anti HIV drug classes, as well as individual drug potency and tolerability has increased, many patients can expect to suppress viral load below the limit of detection in clinical practice, currently defined as 50 copies per milliliter of blood mentioned by CDC (2009) and De Clercq, E. (2009). Even if an effective AIDS vaccine is still lacking, the introduction of HAART greatly extended survival, with reduction of the morbidity and mortality of HIV infection. However in opinion of authors as Carcelain et al (2001) and Bailey et al (2006) with very sensitive methods, a residual viremia is still detected in patients on HAART. Moreover, Desrosiers (1999) and Le Douce et al (2010) suggested that HIV RNA returns to a measurable plasma level in less than two weeks when HAART is interrupted. These observations suggest that even long term suppression of HIV-1 replication by HAART cannot totally eliminate HIV-1. The virus persists in cellular reservoirs because of viral latency, cryptic ongoing replication or poor drug penetration published by Johnston and Barre-Sinoussi (2012) and Brennan et al (2009).

HAART does not completely solve the immune and metabolic alterations during HIV infection. Instead hepatic toxicity from antivirals as zidovudine (AZT) was demonstrated early in the epidemic mentioned by De Clercq (2009). Additional adverse effects and/or regimen adherence difficulties have serious consequences suggested by Johnston and Barre-Sinoussi (2012) such as loss of serum HIV suppression, development of drug-resistant HIV strains, and increased probability of illness progression.

Some anti HIV drugs classes are associated with lactic acidosis, hyperlipidemia, glucose intolerance, diabetes mellitus, fat redistribution and wasting, and atherosclerosis. These features could be related with OS increased by antiretroviral (ART) toxicity which point at the mitochondria as toxic target as suggest Feng et al (2001). It could be produced by a common mechanism through mitochondria dysfunction.

The purpose of this review is to analyze the evidences concerning oxidative metabolism alterations involved in HIV infection. ART toxic mechanism contributing to oxidative 
damage and its association with comorbidities will also be examined. Finally, potential use of antioxidant in HIV naïve and treated patient is also discussed.

\section{Redox Imbalance and HIV Infection}

In the articles by Blocks et al (2002), Kohen and Nyska (2002), Devasagayam et al (2004), and Valko et al (2007) ROS were considered as products of normal physiological processes, involved for instance in the transport of electrons in the mitochondrion and also serve as signaling molecules. The ROS productions are particularly increased in the activated phagocytes and neutrophils representing one part of the microbial activity. In that case, the activation of the immune system may produce a significant local or systemic increase of ROS generation. An excessive amount of these molecules could leads to oxidative modification and, therefore, dysfunction of proteins, nucleic acids, carbohydrates and lipids which were showed by Block et al (2002) and, Kohen and Nyska (2002).The extent of damage that this stress could cause depends on the condition of the antioxidant defence system at the site of inflammation.

In articles by Block et al (2002) and, Kohen and Nyska (2002) ROS are considered as transient, unstable, and largely localized products into cellular compartments, making their direct measurement difficultly. As a result, the role of ROS in pathologic conditions has been inferred from the measurement of indirect markers such as lipid and protein oxidation and the activities of scavenging enzymes.

In a report by Bailey et al (2006) cells of myeloid lineage (e.g., monocytes, macrophages and dendritic cells) are recognized as critical immune cells responsible for a wide range of both innate and adaptative immune functions. They play an important role in the initial HIV infection and therefore contribute to its pathogenesis throughout the course of infection. Infected circulating monocytes are also recruited to the gastrointestinal tract. They later differentiate into macrophages and form the HIV-1 reservoir of the intestine.
Dendritic cells are also involved in the dissemination of HIV-1 following primary infection. After capturing viruses at the site of infection, mature dendritic cells migrate into lymph nodes where they participate in the transmission of HIV-1 to CD4+ T cells. Mature myeloid dendritic cells located in lymph nodes can sustain a very low level virus replication and therefore have a potential role in HIV-1 latency and/or ongoing replication. The mechanism of this viral persistence is not yet known. Macrophages harbouring the CD4 receptor and CCR5 coreceptor are now recognized as early cellular targets for HIV-1. These cells are able to produce and harbour the virus for a longer period. Haematopoitic cells (HPC) have also been proposed to serve as a viral reservoir, since a subpopulation of CD34+ HPCs express CD4 and CCR5 and/or CXCR4 and these cells are susceptible to HIV-1 infection.

Protective mechanisms against pathogens include the generation of ROS whatever their origin, and they may play a role in several cell processes including signalling, proliferation and death.

Almost cells who internalise HIV activate a ROS generating NADPH oxidase enzyme (NOX2,gp91 phox ) and others which in turn contribute to the ROS increase production.

Larbi et al (2007) and also Block et al (2002) proposed that $T$ cell signalling, metabolism and function are influenced by such environmental changes. Because $\mathrm{T}$ cells are present in the inflammatory site they also experiment ROS attack. The ability to cope with ROS-induced OS will depend on protective mechanisms the cell can provide suggested by Valko et al (2007). Mammalian cells are able to activate protective mechanisms to cope with this stress, first by activation specific signal transduction pathway. This involves activation of factor to control transcription of a range of target genes which encode products to mediate biological responses to oxidative stress. Applying low stress levels can result in increased resistance to subsequent otherwise lethal higher stress levels, a process known as hormesis, which is thought to be an adaptation mechanism. 
Chronic immune activation during HIV infection mediates ROS increase production which in turn modulates and activates nuclear transcription factors, which ultimately lead to viral gene expression reported by Gendron et al (2011) and Ma (2010). HIV replicates in activated cells, and there is an intriguing interplay between HIV replication and immune activation. HIVinfected patients have elevated levels of polygonal immunoglobulins demostrated by Le Douce et al (2010).

The pathway involving NF- $\kappa B$ activation in monocyte cells and primary macrophages during HIV-1 infection have been showed by Dröge et al (1991). Persistent HIV-1 infection of macrophages results in increased levels of NF- $\kappa B$ in the nucleus secondary to increased inhibitors ( $\mathrm{I} \kappa \mathrm{B} \alpha$, $\mathrm{I} \kappa \mathrm{B} \beta$, and $\mathrm{I} \kappa \mathrm{B} \varepsilon$ ) degradation, a mechanism postulated to regulate viral persistence. Then, as the synthesis at the transcriptional level proceeds, replication is negatively controlled by Bcl-2 to reach a balance characterized by low virus production and higher Bcl-2 and TRX levels resulting in low but sustained viral production compatible with cell survival.

It has been demonstrated by Gendron et al (2011) that HIV-1 internal ribosome entry site (IRES) located in the 5'unstransleted region is activated during viral infection. OS caused by HIV infection increases the activity of the wild-type IRES. Those distal long terminal repeat (LTR) binding sites upstream of the transcription nuclear factor kappa B (NF- $\mathrm{KB}$ ) binding site are essential for the efficient transcription in monocytes and macrophages. In addition to NF- $\mathrm{KB}$ and Sp1 binding, NF-IL6 and/or USF protein binding to the LTR modulatory region are essential for HIV-1 transcription.

It has been proposed by Larbi et al (2007) that tumour necrosis factor alpha (TNF $\alpha)$ induced NF- $\mathrm{BB}$ activity might be involved in the apoptosis inhibition of macrophage versus $T$ cells in the context of chronic immune activation. Also Ushio (2009), Cross and Templenton (2004) and Trinei et al (2002) reported that the survival mechanism of monocytes and macrophages even if TNF $\alpha$ is best known as a pro- inflammatory mediator capable to induce apoptosis. Cross and Templenton (2004), and Desrosiers (1999) mentioned that HIV1 could manipulate the apoptotic machinery to its advantage inducing a dual regulation of the antiapoptotic protein Bcl-2, resulting in persistent infection of monocytic cells. HIV-1 infection first results in a decrease of Bcl-2 and TRX, permitting an initial boost of replication.

$\mathrm{T}$ cells undergoing many successive cell divisions during adaptive immune responses are subject to proliferative stress reaching limit of replicative senescence. OS in turns lead to expression of Fas/cd95 receptor which reacts with Fas ligand overexpressed too and activates a mechanism of apoptosis for both uninfected and infected CD4+ $\mathrm{T}$ lymphocytes. Those conditions are considered the hallmark of HIV progression suggested by Ma (2010). Authors recognize OS implications in HIV infection related to viral replication despite $\mathrm{CD} 4+\mathrm{T}$ cell apoptosis. Authors as Jaworowsi and Crowe (1999), Mollace et al (2002), Grossman et al (2002), Gendron et al (2011), Ma (2010), Sloand et al (1997) and Zhang et al (2011) provide in vitro evidences in relation to infection-induced OS contribution to CD4+ $\mathrm{T}$ lymphocytes depletion by increasing their rate of apoptosis; particularly by Fas/APO$1 /$ CD95 mechanism. The pro-oxidant stimuli increase the pathogenic effects of HIV which is associated also with a progressive increase of plasma viral load.

Halliwell (2006) showed that enzymatic antioxidants include SOD, catalase (CAT), peroxidase, and some supporting enzymes. The non-enzyme antioxidants can be further classified into directly acting antioxidants (e.g., scavengers and chain breaking antioxidants) and indirectly acting antioxidants (e.g., chelating agents). The former are extremely important as defenses against OS. This subgroup currently contains several hundred compounds. Most of them, including ascorbic and lipoic acids, polyphenols, and carotenoids derived from dietary sources. Also Ushio 2009 proposed that the cell itself synthesizes a minority of these molecules, such as GSH and NADPH. In other report by Valko et al (2007) 
antioxidant vitamins constitute important components of the body's non-enzymatic antioxidant systems. Ascorbic acid (vitamin C), $\alpha$-tocopherol (the principal constituent of vitamin E), and $\beta$-carotene (pro-vitamin A) are the best studied natural antioxidants. The sustained ROS increase generation during HIV infection lead to antioxidant consuming and its mean consequence is damage to cellular components.

Proteins modified by ROS exhibit altered structures, undergo spontaneous fragmentation, manifest increased proteolytic susceptibility, and loss of function. Oxidative protein damage has been found to be high in specific humans regions and to be elevated during aging, neurodegenerative disorders and HIV infection reported by Roberts et al (2010) and Block et al (2002). Phospholipids of biological membranes are highly susceptible to oxidation by ROS and also are involved in the process known as lipid peroxidation. This is a free radical mediated pathway and its final products are used as an index of irreversible damage of cell membrane phospholipids published by Roberts et al (2010) and Block et al (2002). Oxidative modification to DNA and its consequences such as gene mutation and DNA deletions have long been implicated in the pathogenesis of a variety of human disorders associated with mitochondrial dysfunction and aging showed by Devasagayam et al (2004). Oxidative damage to DNA can be variable, producing structural injure such as strand breaks, protein/DNA cross-links, and/or modification of base pairs.

OS is thought to be responsible, at least in part, for cellular senescence. Aging is associated with a loss in protective immunity mainly characterized by poor $\mathrm{T}$ cell responses to stimulation, low responses to vaccination and accumulation of CD8 cells which seem to be in a state of replicative senescence. Several hypotheses have been proposed by Roberts et al (2010), Block et al (2002), Kregel and Zhang, (2007) and Tchkonia et al (2010) to explain this, with the role of chronic proliferative and OS featuring as a major factor.
Several studies by Sonnerborg et al (1988), Pasupthi et al (2009), Coaccioli et al (2010), Awodele et al (2012), Redhage et al (2009), Wanchu et al (2009) and Stephensen et al (2007) found that both asymptomatic HIV-infected individuals and AIDS patients had higher levels of OS, as indicated by increased plasma metabolites of lipid peroxidation and/or reduced antioxidant levels, compared with healthy controls. A reduced plasma GSH and total antioxidant concentration, increased MDA and peroxides concentrations and altered CAT and SOD activity have been demonstrated. These findings could be explained in part by several mechanisms such as low intake of antioxidant or their precursors, malabsorption and, in peripheral tissue, enhanced cysteine metabolism with a consequent loss of sulphur-group may account for GSH and antioxidant deficiency during HIV infection. Abnormally high levels of ROS as a consequence of chronic immune system activation by HIV infections have been proposed by Pasupthi et al (2009) and suggest it could lead to a decline of antioxidants defence molecules and accumulative damage of cellular components generating augmented lipid peroxidation products, oxidized proteins and altered DNA sequences. Almost redox implicated enzymes and molecules physiologically endogenous generated are involved in detoxification and general metabolism which could be altered too. Guaraldi et al (2011) and Gil (2011) argue that persistent OS have a dramatic impact on immunological, clinical and nutritional status in HIV infection. Romero-Avila and Roche (1998) Dröge et al (1991) and Capeau (2011) recognized that OS caused by the imbalance between the generation and detoxification of ROS, could be related to premature aging, dementia, and other related adverse conditions and comorbidities during HIV infection.

Observations that direct oxidant challenge mimics many of the cellular and transcriptional change seen with aging also strengthen the link between OS and the rate of aging. The increase ROS generation at a given anatomical site may cause a redox mediated dysregulation at multiple and 
distant anatomical sites of the organism suggested by Droge (2002) and M (2010). Major chronic disease such as cancer, cardiovascular disorder, neurodegeneration, and waste syndrome incidence increase rapidly in HIV patients and also resound in mortality have been observed in first years of the infection (natural infection). These observations support the notion of OS as a common underlying mechanism for premature aging.

\section{Antiretroviral Treatment in HIV}

ARV therapy in HIV-infected individuals has changed dramatically over the past several years, from monotherapy in the 1990s to the current use of combination regimens of different class of anti HIV drugs referred by Reports of CDC (2009).

ART induces meanly a biphasic decline of HIV-1 RNA with a rapid decline of infected CD4+ T cells (half life 0.5 day) followed by a decline originating from infected tissue macrophages (half life 2 weeks) reported by Hammer et al (2008).

UNAIDS epidemic update (2011) and De Clerq (2009) mentioned that Nucleoside Reverse Transcriptase inhibitors (NRTIs), found to be effective in inhibiting the replication of HIV. Azidothymidine (AZT, zidovudine) was the first (1987) of a long list of 2',3-dideoxynucleoside analogues as ddI (didanosine), ddC (zalcitabine), d4T (stavudine), 3TC (lamivudine), abacavir (ABC), and emtricitabine ( $(-)$ FTC) which were introduced in HIV treatment.

All NRTIs have in common that they have to be phosphorylated intracellularly, successively to the 5'mono-, 5'-di- and 5'triphosphate, before the latter, can interact with the HIV reverse transcriptase (RT). As an alternate substrate/competitive inhibitors act as obligatory chain terminators. In this form they compete with any of the natural substrates (dTTP, dCTP, dATP or dGTP) for the HIV RT reaction. The initial wave started by the description of AZT, still continues to stimulate new attempts to launch new NRTIs.
Nucleotide reverse transcriptase inhibitors (NtRTIs) should be considered as nucleotide analogues that contain a phosphonate moiety instead of the readily hydrolysable ester linkage present in the nucleotide form in which the NRTIs are finally incorporated into the DNA. The NtRTIs belong to the acyclic nucleoside phosphonates (ANPs). Two of those ANPs are currently on the market as NtRTIs: adefovir (PMEA) and tenofovir [(R)-PMPA]. Like the NRTIs, the NtRTIs eventually act as obligatory chain terminators in competition with dATP, but the NtRTIs need only two phosphorylations to be converted to their active (diphosphate) form, since they are already phosphonylated in their parental form. When it's finally incorporated as chain terminator into the DNA, they are incorporated via this phosphonate group which makes their excision by exonucleases more difficult than if they were incorporated via the readily cleavable phosphate group.

Also UNAIDS epidemic update (2011) and De Clerq (2009) published that the target for the anti-HIV-1 action of the non nucleoside reverse transcriptase inhibitors (NNRTIs) was identified as an allosteric site, distinct, but a close distance, from the catalytic site. Nevirapine (Viramune1), delavirdine (Rescriptor1), efavirenz (Sustiva1, Stocrin1) and etravirine (Intelence1) are available at market.

In CDC report (2009) and De Clerq (2009) protease inhibitors (PIs) were considered those compounds which can replace a peptide linkage [- $\mathrm{NH}-\mathrm{CO}-]$ by an hydroxyethylene group $[-\mathrm{CH} 2-\mathrm{CH}(\mathrm{OH})-$ ] and saquinavir was the first PI to be 'rationally' designed on this conceptual base. Then followed, all based on the same principle, ritonavir, indinavir, nelfinavir, amprenavir, lopinavir, atazanavir, fosamprenavir and darunavir; they all contain a central 'core' motif existing of a hydroxyethylene scaffold. The only exception to the rule is tipranavir which is based on a coumarin scaffold. PIs have been shown to fit snugly inside the active site of the dimeric aspartyl HIV protease. 
There is actually one fusion inhibitor (FI) currently available for the treatment of HIV infections, and this is enfuvirtide (Fuzeon1), a polypeptide of 36 amino acids that is homologous to, and engages in a coil-coil interaction with the heptad repeat HR regions of the viral glycoprotein gp41 As it is not orally bioavailable, it must be injected parenterally (subcutaneously) twice daily.

Co-receptors inhibitor (CRIs) interact with the co-receptors CCR5 or CXCR4 used by, respectively, $\mathrm{M}$ (macrophage)- tropic and $\mathrm{T}$ (lymphocyte)-tropic HIV strains (now generally termed R5 and X4 strains, respectively) to enter the target cells. Within the whole virus cell entry process, interaction of the viral glycoprotein gp 120 with the co-receptor occurs after the interaction of the viral glycoprotein gp120 with the CD4 receptor and before fusion of the viral glycoprotein gp41 with the outer cell membrane. There is, at present, only one CRI available (licensed in 2007 for clinical use), which is the CCR5 antagonist maraviroc (Selzentry1) mentioned by UNAIDS report (2011).

The first integrase inhibitor (INIs), raltegravir has been approved in 2007 for clinical use. The HIV integrase has essentially two important catalytic functions (30-processing and strand transfer). Raltegravir is targeted at the strand transfer reaction and it has proven highly effective in reducing viral loads in HIV-infected patients.

De Clerq (2009) showed that ART treatment options are continually being updated because of availability of new medications, formulations, and dosing recommendations. Synergy, lower toxicity and prevention of drug resistance development have remained the main reasons for combining different anti-HIV drugs in the treatment of AIDS. Different classes of HIV inhibitors available i.e. NRTIs, NtRTIs, NNRTIs, FIs, CRIs and INIs, has increased far beyond any reasonable practicability the number of possible drug combinations.

HAART has been associated with increased survival, reduction in opportunistic infections, improved growth and neurocognitive function, and improved quality of life. In others sense HAART produces maximal suppression of viral replication to prevent disease progression, preserve immunological function, and reduce the development of resistance.

In UNAIDS report (2011) is reported that the optimal timing of HAART initiation is unknown and continues to be controversial. Current trends suggest movement toward earlier treatment in adults that will likely influence recommendations for initiation in children.

The current recommendation for initial HAART is to use 3 drugs from 2 classes of drugs: a backbone of 2 NRTI combined with either a NNRTI or a PI. The optimal regimen depends on multiple factors including age; availability, and palatability of appropriate drug formulations; the potency, complexity, and toxicity of the regimen. Combinations of anti-HIV drugs are used during HIV infection evolution as clinical guidelines re- commend.

In a research study by Bailey et al (2006) different reservoirs for virus persist might proposal: in anatomical sanctuary sites where drug penetration or potency is suboptimal; as integrated but transcriptionally silent provirus, maintained in long-lived cells or by homeostatic proliferation; or by low levels of ongoing replication that are incompletely suppressed by HAART.

Anatomical sanctuary sites as a source of viral rebound were also briefly considered by Desrosiers (1999). Tissue levels of ART are considerably lower, especially in the brain and such tissues as lymph nodes than those in plasma. It is possible, then, that patients might experience ongoing viral replication in tissues even when it is not observed in blood. One argument against sanctuary sites as a location of ongoing replication and source of viral rebound is the ability of regimens that do not cross the blood-brain barrier very well to nonetheless suppress the virus below the limit of detection. 


\section{Toxicity during Antiretroviral Treatment and Associated Diseases. Clinical Evidences}

Roberts et al (2010) and Sahnoun et al (1997) showed that mitochondria are present in every eukaryotic cell (from hundreds to thousands) with the exception of peripheral red blood cells. It is believed that human cells can produce $\sim 10$ million ROS / mitochondrion / day. Mitochondrial ROS are produced during oxidative metabolism through the one electron reduction of oxygen $\left(\mathrm{O}_{2}\right)$ to form $\mathrm{O}_{2}{ }^{-}$-. The mitochondrial electron transport chain generates $\mathrm{O}_{2}{ }^{\circ}$ - predominantly at complexes I and III. ROS originating from mitochondria are thought to be important in both physiological (modulating cell differentiation and function) and pathological processes.

Kohen and Nyska (2002) suggested a variety of cellular systems including NADPH oxidase, xanthine oxidase, uncoupled endothelial nitric oxide syntase (eNOS) and cytochrome p450 enzymes which can generate ROS also, but in most mammalian cells, mitochondria are the principal organelles for ROS production.

Mitochondria contain a DNA-pool (mtDNA) distinct from nuclear DNA. DNA polymerase- $\gamma$ is the enzyme that replicate and maintains mtDNA. The proteins encoded by mtDNA are involved in electron transport complexes of oxidative phosphorylation (OXPHOS). Phosphorylated- NRTI inhibition $\gamma$-DNA polymerase has been argued by Lee et al (2003), Coté et al (2003), Lagathu et al (2007), Caron et al (2008) and López et al (2002). This inhibition results in mtDNA depletions, altered in OXPHOS proteins synthesis, energy deprivation and tissues dysfunction. All contribute to increase the formation of ROS and respected OS.

The OS effects due to phophorylated-NRTI mitochondrial toxicity may amplify some of the pathophysiology and phenotypic events in infection are proposed by Hulgan et al (2003), Miró et al (2005), Mallon (2007), Caron et al (2010), Lewis et al (2001), Day and Lewis (2004) and Masiá et al (2007). This aspect result in a new tissue target as treatment is prolonged with increased longevity from AIDS, resistance to NRTI appears, and AIDS become a relatively manageable chronic illness.

One of the most vexing problems that have appeared in the HAART era is the occurrence of the HIV-associated lipodystrophy syndrome, the hallmarks of which are body shape and metabolic abnormalities that are likely to be multifactorial respect its origin. Mallon (2007), Miro et al (2005), Milazzo et al (2010) and Vassimont et al (2010) mentioned that the body shape abnormalities in HIV evolution include subcutaneous fat atrophy and visceral or central fat accumulation. The metabolic abnormalities include elevations in serum triglycerides and total cholesterol, suppression of serum high-density lipoprotein cholesterol levels, and hyperinsulinemia in normoglycemic individuals. There are also reports of chronic asymptomatic elevations in serum lactate (probably associated with the use of NRTIs) and decreases in bone density.

It has been proposed that adverse effects to ARV (including myopathy, cardiomyopathy, anaemia, hyperlactataemia/ lactic acidosis, pancreatitis, polyneuritis and lipodystrophy) could be mediated by mitochondrial (mt) toxicity. Several studies relating OS with HAART associated to mitochondrial dysfunction were published by Masiá et al (2007), Ngondi et al (2006), Mandas et al (2009), Gil et al (2011), Lagathu et al (2007) and Sundaram et al (2008). Although the relation is proposed there is still a debate about whether it plays a direct role in HAART toxicity. The mechanism is unclear. DNA pol $\gamma$ inhibition favours to an energy deprivation and increase ROS formation. OS-mediated cell damage results in part via ROS reactions mainly due to NRTI. This antiviral class has been implicated as a cause of several insidious and sometimes irreversible chronic toxicities demonstrated by Vassimon et al (2010). 
Reports by Gil et al (2011), Masiá et al (2007) and Ngondi et al (2006) about HAART influences on OS indexes noticed a decrease in the antioxidant system, an increase in damaged molecules and a failure to repair oxidative damage. Ngondi et al (2006) assessed the effect of different HAART combinations in OS parameters and found an increase in lipid oxidation while antioxidants decrease. They showed significant differences in lineal association between some parameters as sulphydryls and albumin with CD4+ T lymphocytes count and with viral load.

Hulgan et al (2003) quantified plasma F2 isoprostanes as OS index. They planned different strategies and found that this index augmented in $120 \mathrm{HIV}+$ individuals treated with HAART but they could not distinguish differences due to drugs or HAART combinations. During long term follow-up Hulgan et al (2006) did not found association with peripheral neuropathy or cardiovascular risk in their studies context. Meanwhile Masiá et al. (2007) found increased plasma peroxide associated with established cardiovascular risk factors in the study context.

Previous work by McComsey et al (2005) describe some clinical and metabolic benefits during replacement of antiviral treatment while others by Milazzo et al (2010), Yousefi et al (2011), Hurwitz et al (2007) and Gil et al (2005a) found clinical and metabolic benefits during dietary micronutrient intake improvement in treatment or naive patients. For several years, the attention of different research groups as Aukrust et al (2003), Hulgan et al (2006) and Hurwitz et al (2007) has been largely focused on damages induced by ART on mtDNA and on the consequence of its depletion. Reports show that OS caused by NRTIs can play an important role in the pathogenesis of lipodystrophy, and that part of the response (i.e., changes of gene expression that can be observed in adipose tissue) is not due to mtDNA depletion per se but to the response to ROS increase.

The accumulation of oxidized mitochondrial proteins, typically observed with physiological aging mentioned by Lee and
Wei (2007) or in age-related disorders reported by Block et al (2002), could occur at higher rate in lipodystrophy, and could significantly contribute to the pathogenesis of such a side effect of ART.

\section{Factors to be Considered during Antiviral Treatment Concerning Oxidative Stress Condition}

When patients are diagnosed as HIV+, therapy is initiated based on clinical assessment, CD 4 + cell count and viral load and also are considered if HAART should be deferred. The HAART combinations applied vary depending on therapeutics objectives, cost and availability on the market as recommended by UNAIDS (2008) and WHO reports (2011).

Apart from the toxic effects of ART, the development of new strains of the virus and subsequently resistance to available medications, especially in areas with acceptable coverage of the therapy, indicate that in addition to ART, other alternative therapies are needed in this regard as proposed Aquaro et al (2008) and Edeas et al (2010).

Researches by Gil et al (2005), Milazzo et al (2010), Mandas et al (2009) and Sundaram et al (2008) showed that highly oxidative state during the progress of HIV infection can be promoted by the host. Thus, treating the patients with antioxidants since early stages of the disease is believed to be beneficial argued by Tang et al (2005), Gil et al (2005a) and Hurwitz et al (2007).

Twenty-five years ago antioxidants are recognized that could influence on a disease process if ROS caused or significantly contributed to the progression of the disease published by Edeas (2009), Edeas et al (2010) and Gil et al (2005). Then, and now evidences support the view that increased ROS formation is usually a consequence of tissue damage by a disease or toxin. Sometimes it contributes significantly, more often it does not and, in some cases ROS may be exerting a protective role, e.g. by down-regulating inflammation. Under such circumstances, assuming that an administered antioxidant 
could reach the site of increased ROS formation, it would at best marginally affect secondary tissue damage and could even be deleterious. Sometimes pro-oxidants might be more beneficial as suggested Ma (2010) and Milazzo et al (2010). Indeed, flavonoids may act just as much as pro-oxidants in the gastrointestinal tract and in cell culture systems as they do as antioxidants.

Theoretical an OS enhance occurs as a dynamic process of immune reconstitution, too. In most of HIV patients the treatment aiming to reduce and control viral replication could produce clinical deterioration as newly inflammatory occurs when HIV becomes a chronic condition. Immune reconstitution is related to lymphocytes redistribution, systemic immune activation changes, modification of lymphocytes turnover rate, and others events proposed by Aquaro et al (2008) and, Kohen and Nyska (2002). This hypothesis will require larger prospective studies including immunological assessments.

Long term clinical implications of OS and how it is related to ART therapy associated disease should be studied. The oS evaluations will therefore become potential utility factors to follow antiviral combinations effects, as well as the usefulness of antioxidant and alternative therapies. It is important during the antioxidant and micronutrient supplementation recognize between nutritional incidence of these factors for immune response as its use as preventive therapy. In terms of which the administration of vitamin and multifunctional antioxidant become advisable before initiation and during ART treatment.

Clinical trials have been conducted to determine vitamin supplements with antioxidant action effect on HIV infection. Some experimental and epidemiological studies indicate beneficial effects of antioxidant vitamin supplementation on the progression of HIV infection reported by Milazzo et al (2010), Yousefi et al (2011) and Hurwitz et al (2007).
Multivitamin supplementation, but not vitamin A with in certain limit could produces some alteration related to its accumulation in body, did appear to confer some benefits on HIV-infected women and their offspring. Multivitamin supplementation was associated with increased CD4+ cell counts, more weight gain during the third trimester of pregnancy, and improved birth outcomes (including higher birth weights and lower infant mortality). In populations of HIVinfected patients treated with HAART, the role of OS in disease progression has become more complicated. Whereas HIV itself increases OS levels through replication, control of the virus with ART therapy may not, as one might expect, reduce oxidative damage levels, as the medications themselves may increase OS mentioned by Feng et al (2001) and Carcelain et al (2001).

Micronutrients play a critical role in the maintenance of immune function (including mucosal immunity) and overall metabolism. With the complexity of HIV infection and treatment, and the pace of clinical research, it is not surprising that sophisticated studies of the role of micronutrients in HIV have lagged somewhat behind other areas of inquiry. Much credit is due to researchers who have been asking, and continue to ask, questions about micronutrient status and the progression of HIV infection. As HIV becomes a more chronic, manageable disease, and treatment becomes available to more of those infected throughout the world, it may be possible to begin to more precisely define the areas in which micronutrients may help to maximize the clinical status of HIV-infected patients. Studies to date reveal that this is a complex topic. For example, it is difficult to ascertain true micronutrient status, measures of intake are imprecise, recommendations for desired intakes vary, and surrogate markers of OS and strategy to valuate its references and deviation differ from one author to another. For HIV-infected individuals with adequate viral suppression, but inadequate CD4+ cell counts, micronutrients could play a role in boosting the immune response demostrated by Valko et al (2007) and Tang et al (2005). 
As HIV-infected individuals co-exist with chronic viral infection and chronic ART therapy that in turn increase OS, the need to examine creative interventions to minimize long-term complications such as fat atrophy, insulin resistance, lipid abnormalities are becoming increasingly important. The role that micronutrients with antioxidants potential, may play in modulating these toxicities is not yet clear and should be studied. In addition, for the patient with multiple chronic viral infections, such as HIV and hepatitis B and/or C, micronutrient supplementation may also be beneficial in minimizing the comorbidities associated with these coinfections. As the HIV-infected population ages, and the risk of cardiovascular disease increases, the general strategy and combination of the OS counteracts interventions represent an important complementary deal for HIV infection treatment.

In conclusion, the knowledge on HIV physiopathology in general and the recognition of OS implications on it, has gained tremendously advances. This has led to improvements in specific and alternative treatments which influences on declines in HIV morbidity and mortality. The ability to combine diagnostic indexes in order to integral view approach represent valuable tools both for understanding the pathogenic actions of the virus and for the clinical monitoring of HIV-infected patients. The optimal use of these tools in the clinical setting, however, still remains to be defined. Beneficial of counteract OS strategy have to be better designed and proved in terms to contribute to ameliorate morbidity and improve quality of life in HIV infection.

\section{References}

Aquaro, F., Scopelliti, F., Pollicita, M. \& Perno, C. F. (2008). "Oxidative Stress and HIV Infection: Target Pathways for Novel Therapies?," Future HIV Therapie, 2 (4), 327-338.

Aukrust, P., Muller, F., Svardal, A. M., Ueland, T., Berge, R. K. \& Froland, S. S. (2003). "Disturbed Glutathione Metabolism and Decreased Antioxidants Levels in Human
Immunodeficiency Virus-Infected Patients during Highly Active Antiretroviral Therapy-Potential Immunomodulatory Effects of Antioxidants," Journal Infection Disease, 188, 232-238.

Awodele, O., Olayemi, S. O., Nwite, J. A. \& Adeyemo, T. A. (2012). "Investigation of the Levels of Oxidative Stress Parameters in HIV and HIV-TB Co-Infected Patients," Journal of Infection in Developing Countries, 6 (1), 7985.

Bailey, J. R., Sedaghat, A. R., Kieffer, T., Brennan, T., Lee, P. K., Wind-Rotolo, M., Haggerty, C. M., Kamireddi, A. R., Liu, Y., Lee, J., Persaud, D., Gallant, J. E., Cofrancesco, J., Quinn, T. C., Wilke, C. O., Ray, S. C., Siliciano, J. D., Nettles, R. E. \& Siliciano, R. F. (2006). "Residual Human Immunodeficiency Virus Type 1 Viremia in Some Patients on Antiretroviral Therapy Is Dominated by a Small Number of Invariant Clones Rarely Found in Circulating CD4+ T Cells," Journal of Virology, 80(13), 6441-6457.

Block, G., Dietrich, M., Norkus, E. P., Morrow, J. D., Hudes, M., Caan, B. \& Packer, L. (2002). "Factors Associated with Oxidative Stress in Human Populations," American Journal of Epidemiology, 156(3), 274-285.

Brennan, T. P., Woods, J. O., Sedaghat, A. R., Siliciano, J. D., Siliciano, R. F. \& Wilke, C. O. (2009). "Analysis of Human Immunodeficiency Virus Type 1 Viremia and Provirus in Resting CD4+ T Cells Reveals a Novel Source of Residual Viremia in Patients on Antiretroviral Therapy," Journal of Virology, 83(17), 8470-8481.

Capeau, J. (2011). "Premature Aging and Premature Age-Related Comorbidities in HIV-Infected Patients: Facts and Hypotheses," Clinical Infectious Diseases, 53(11), 1120 -1126.

Carcelain, G., Debre, P. \& Autran, B. (2001). "Reconstitution of CD4+ T Lymphocytes in HIV-Infected Individuals following Antiretroviral Therapy," Current Opinion in Immunology, 13, 483-488.

Caron-Debarle, M., Boccara, F., Lagathu, C., Antoine, B., Cervera, P., Bastard, J.- P., 
Vigouroux, C. \& Capeau, J. (2010). "Adipose Tissue as a Target of HIV-1 Antiretroviral Drugs. Potential Consequences on Metabolic Regulations," Current Pharmaceutical Design, 16 (30), 3352-3360.

Caron, M., Auclairt, M., Vissian, A., Vigouroux, C. \& Capeau, J. (2008). "Contribution of Mitochondrial Dysfunction and Oxidative Stress to Cellular Premature Senescence Induced by Antiretroviral Thymidine Analogues," Antiviral Therapy, 13(1), 27-38.

Centres for Disease Control and Prevention. Guidelines for Prevention and Treatment of Opportunistic Infections in HIV-Infected Adults and Adolescents. MMWR 58 (No. RR4) April 10, 2009. [AIDSinfo Web Site] Available at: http://www.aidsinfo.nih.gov/Guidelines

Coaccioli, S., Grapa, G., Fantera, M., Del Giorno, R., Lavagna, A., Standoli, M. L., Frongillo, R., Biondi, R. \& Paxeddu, A. (2010). "Oxidant/Antioxidant Status in Patients with Chronic HIV Infection," Clinical Therapy, 161(1), 55-58.

Cote, H. C. F., Yip, B., Asselin, J. J., Chan, J. W., Hogg, R. S., Harrigan, P. R. O., Shaughnessy, M. V. \& Montaner, J. S. G. (2003). "Mitochondrial/Nuclear DNA Ratios in Peripheral Blood Cells from Human Immunodeficiency Virus (HIV)-Infected Patients Who Received Selected HIV Antiretroviral Drug Regimens," Journal of Infection Disease, 187, 1972-1976.

Cross, J. V. \& Templeton, D. J. (2004). "Thiol Oxidation of Cell Signalling Proteins: Controlling an Apop Totic Equilibrium," Journal of Cell Biochemistry, 93, 104 -111.

Day, B. J. \& Lewis, W. (2004). "Oxidative Stress in NRTI-Induced Toxicity," Cardiovascular Toxicology, 4, 207-216.

De Clercq, E. (2009). "The History of Antiretrovirals: Key Discoveries over the Past 25 Years," Reviews in Medical Virology, 19, 287-99.

Desrosiers, R. C. (1999). "Strategies used by Human Immunodeficiency Virus That Allow
Persistent Viral Replication," Natural Medicine, 5, 723-725.

Devasagayam, T. P. A., Tilak, J. C., Boloor, K. K., Sane, K. S., Ghaskadbi, S. S. \& Lele, R. D. (2004). "Free Radicals and Antioxidants in Human Health: Current Status and Future Prospects," Journal Association of Physicians India, 52, 794 -803.

Dröge, W., Eck, H. P., Gmünder, H. \& Mihm, S. (1991). "Requirement for Prooxidant and Antioxidant States in $\mathrm{T}$ cell Mediated Immune Responses - Relevance for the Pathogenetic Mechanism of AIDS?," Journal of Molecular Medicine, 69(21), 1118-1122.

Edeas, M. (2009). "Anti-Oxydants, Controverses et Perspectives: Comment Expliquer L'echec," Journe De Societe Biologe, 203(3), 271-280.

Edeas, M., Attaf, D., Mailfert, A. S. Nasu, M. \& Joubet, R. (2010). "Reaction, Mitochondria and Oxidative Stress: Potential Role of Antioxidants," Pathologie Biologie, 58, 220225.

Feng, J. Y., Johnson, A. A., Johnson, K. A. \& Anderson, K. S. (2001). "Insights into the Molecular Mechanism of Mitochondrial Toxicity by AIDS Drugs," Journal of Biology and Chemistry, 276, 23832-23837.

Gendron, K., Ferbeyre, G., Heveker, N. \& Brakier, G. (2011). "The Activity of the HIV-1 IRES Is Stimulated by Oxidative Stress and Controlled by a Negative Regulatory Element," Nucleic Acid Research, 39 (3), 902-912.

Gil, L., Lewis, L., Martinez, G., Tarinas, A., González I., Alvarez, A., Tápanes, R., Giuliani, A., León, O. S. \& Pérez, J. (2005a). "Effect of Increase of Dietary Micronutrient Intake on Oxidative Stress Indicators in HIV/AIDS Patients," International Journal of Vitamin and Nutrition Research, 75, 19-27.

Gil, L., Martínez, G., González, I., Alvarez, A., Molina, R., Tarinas, A., León, O. S. \& Pérez, J. (2003). "Contribution to Characterization of Oxidative Stress in HIV/AIDS Patients," Journal of Pharmacological Research, 47, 217-224. 
Gil, L., Pérez, D., Tápanes, R. \& Grune, T. (2005). "Does Mitochondrial Dysfunction during Antiretroviral Therapy in Human Immunodeficiency Virus Infection Suggest Antioxidant Supplementation as a Beneficial Option?," Redox Report, 10, 113-119.

Gil, L., Tarinas, A., Hernández, D., Riverón, V. B., Pérez, D., Tápanes, R., Capó, V. \& Pérez, J. (2011). "Altered Oxidative Stress Indexes Related to Disease Progression Marker in Human Immunodeficiency Virus Infected Patients with Antirretroviral Therapy," Journal of Biomedicine and Aging Pathology, 1 (1), 8-15.

Gild el Valle, L. (2011). Chapter 2: Pathophysiological Implications of Altered Redox Balance in HIV/AIDS Infection: Diagnosis and Counteract Intervention. In "Oxidative Stress: Diagnostics, Prevention, and Therapy". 39 -70. Editors: Andreescu S, Hepel M. ISBN13: 9780841226838

Grossman, Z., Meier-Schellersheim, M., Sousa, A. E., Victorino, R. M. M. \& Paul, W. E. (2002). "CD4+ T-Cell Depletion in HIV Infection: Are We Closer to Understanding the Cause?," Nature Medicine, 8 (4), 319323.

Guaraldi, G., Orlando, G., Zona, S., Menozzi, M., Carli, F., Garlassi, E., Berti, A., Roverato, A. \& Palella, F. (2011). "Premature AgeRelated Comorbidities among HIV-Infected Persons Compared with the General Population," Clinical Infection Disease, 53(11), 1120-1126.

Halliwell, B. (2006). "Reactive Species and Antioxidants. Redox Biology is a Fundamental Theme of Aerobic Life," Plant Physiology, 141, 312-322.

Hammer, S. M., Eron, J., Reiss, P., Schooley, R. T., Thompson, M. A., Walmsley, S., Cahn, P., Fischl, M. A., Gatell, J. M. \& Hirsch, M. S. (2008). "Antiretroviral Treatment of Adult HIV Infection: Recommendations of the International AIDS Society-USA Panel," Journal of the American Medical Association, 300 (5), 555.

Hulgan, T., Hughes, M., Sun, X., Smeaton, L. M., Terry, E., Robbins, G. K., Shafer, R. W.,
Clifford, D. B., McComsey, G. A. \& Canter, J. A. (2006). "Oxidant Stress and Peripheral Neuropathy during Antiretroviral Therapy: An AIDS Clinical Trials Group Study," Journal of Acquired Immune Deficiency Syndrome, 42, 1-5.

Hulgan, T., Morrow, J. D., D'Aquila, R. T., Raffanti, S., Morgan, M., Rebeiro, P. \& Haas, D. W. (2003). "Oxidant Stress is Increasing during Treatment of Human Immunodeficiency Virus Infection," Clinical Infection Disease, 37, 1711-1717.

Hurwitz, B. E., Klaus, J. R., Llabre, M. M., Gonzalez, A., Lawrence, P. J., Maher, K. J., Greeson, J. M., Baum, M. K., Shor-Posner, G. \& Skyler, J. S. (2007). "Suppression of Human Immunodeficiency Virus Type 1 Viral Load with Selenium Supplementation," Archives of International Medicine, 167, 148-154.

Jaworowsi, A. \& Crowe, S. M. (1999). "Does HIV Cause Depletion of CD4+ T Cells In Vivo by the Induction of Apoptosis?," Immunological Cell Biology, 77, 90-98.

Johnston, R. \& Barre-Sinoussi, F. (2012). "Controversies in HIV Cure Research," Journal of the International AIDS Society, 15, 16.

Kohen, R. \& Nyska, A. (2002). "Oxidation of Biological Systems: Oxidative Stress Phenomena, Antioxidants, Redox Reactions, and Methods for Their Quantification," Toxicological Pathology, 30, 620- 650.

Kregel, K. C. \& Zhang, H. J. (2007). "An Integrated View of Oxidative Stress in Aging: Basic Mechanisms, Functional Effects, and Pathological Considerations," American Journal Physiology Regulatory Integral Composition Physiology, 292, 18-36.

Lagathu, C., Eustace, B., Prot, M., Frntz, D., Gu, Y., Bastard, J. P., Maachi, M., Azoulay, S., Briggs, M., Caron, M. \& Capeau J. (2007). "Some HIV Antiretrovirals Increase Oxidative Stress and Alter Chemokine, Cytokine or Adiponectin Production in Human Adipocytes and Macrophages," Antiviral Therapy, 12, 489-500. 
Larbi, L., Kempf, J. \& Pawelec, G. (2007). "Oxidative Stress Modulation and $\mathrm{T}$ Cell Activation," Experimental Gerontology, 42, 852-858.

Le Douce, V., Herbein, G., Rohr, 0. \& Schwartz, C. (2010). "Molecular Mechanisms of HIV-1 Persistence in the Monocyte-Macrophage Lineage," Retrovirology, 7, 32.

Lee, H. C. \& Wei, Y. H. (2007). "Oxidative Stress, Mitochondrial DNA Mutation, and Apoptosis in Aging," Experimental Biological Medicine, 232, 592-606.

Lee, H., Hanes, J. \& Johnson, K. A. (2003). "Toxicity of Nucleoside Analogues Used to Treat AIDS and the Selectivity of the Mitochondrial DNA Polymerase," Biochemistry, 42, 14711-14719.

Lewis, W., Copeland, W. C. \& Day, B. J. (2001). "Mitochondrial DNA Depletion, Oxidative Stress and Mutation: Mechanism of Nucleoside Reverse Transcriptase Inhibitor Toxicity," Laboratory Investigation, 81, 777-790.

López, S., Miro, Ò. \& Beato, A. (2002). 'Assessment of Mitochondrial Toxicity of Diverse Highly Active Antiretroviral Therapy Regimens by a Simultaneous Genetic and Biochemical Approach,' Antiviral Therapy, 7, 15.

Ma, O. (2010). "Transcriptional Responses to Oxidative Stress: Pathological and Toxicological Implications," Pharmacology \& Therapeutics, 125, 376-393.

Mallon, P. W. (2007). "Pathogenesis of Lipodystrophy and Lipid Abnormalities in Patients Taking Antiretroviral Therapy," AIDS, 9, 3-15.

Mandas, A., Lorio, E. L., Congiu, M. G., Balestrieri, C., Mereu, A., Cau, D. \& Curreli, N. (2009). "Oxidative Imbalance in HIV-1 Infected Patients Treated with Antiretroviral Therapy," Journal of Biomedicine And Biotechnology, 18, 67- 79.

Masiá, M., Padilla, S., Bernal, E., Almenar, M. V., Molina, J., Hernández, I., Graells, M. L. \&
Gutiérrez, F. (2007). "Influence of Antiretroviral Therapy on Oxidative Stress and Cardiovascular Risk: A Prospective Cross-Sectional Study in HIV-Infected Patients," Clinical Therapy, 29, 448-455.

Mccomsey, G. A., Paulsen, D. M., Lonergan, J. T., Hessenthaler, S. M., Hoppel, C. L., Williams, V. C., Fisher, R. L., Cherry, C. L., White-Owen, C., Thompson, K. A., Ross, S. T., Hernandez, J. E. \& Ross, L. L. (2005). "Improvements in Lipoatrophy, Mitochondrial DNA Levels and Fat Apoptosis after Replacing Stavudine with Abacavir and Zidovudina," AIDS, 10, 15-23.

Milazzo, L., Menzaghi, B., Caramma, I., Nasi, M., Sangaletti, O., Cesari, M., Poma, B. Z., Cossarizza, A., Antinori, S. \& Galli, M. (2010). "Effect of Antioxidants on Mitochondrial Function in HIV-1-Related Lipoatrophy: A Pilot Study," AIDS, 26(11), 1207-1214.

Miró, O., López, S., Cardellach, F. \& Casademont, J. (2005). "Mitochondrial Studies in HAART-Related Lipodystrophy: From Experimental Hypothesis to Clinical Findings," Antiviral Therapy, 10 (2), 73-81.

Mollace, V., Salvemini, D., Riley, D., PMuscoli, C., Iannone, M., Granato, T., Masuelli, L., Modesti, A., Rotiroti, D. \& Nistico, R. (2002). "The Contribution of Oxidative Stress in Apoptosis of Human Cultured Astroglial Cells Induced by Supernatants of HIV-1Infected Macrophages," Journal of Leukocyte Biology, 71, 65-72.

Ngondi, J. L., Oben, J., Forkah, D. M., Etame, L. H. \& Mbanya, D. (2006). "The Effect of Different Combination Therapies on Oxidative Stress Markers in HIV Infected Patients in Cameroon," AIDS Research Therapy, 3, 19-26.

Pasupthi, P., Ramachandran, T., Sindhu, P. J., Saravanan, G. \& Bakthavathsalam, G. (2009). "Enhanced Oxidative Stress Markers and Antioxidant Imbalance in HIV Infection and Aids Patients," Journal of Science Research, 1, 370-380.

Redhage, L. A., Shintani, A., Haas, D. W., Emeagwali, N., Markovic, M., Oboho, I., Mwenya, C., Erdem, H., Acosta, E. P., Morrow, J. D. \& Hulgan, T. (2009). "Clinical Factors 
Associated with Plasma F2-Isoprostane Levels in HIV-Infected Adults," HIV Clinical Trials, 10, 181-192.

Roberts, R. A., Smith, R. A., Safe, S., Szabo, C., Tjalkens, R. B. \& Robertson, F. M. (2010). "Toxicological and Pathophysiological Roles of Reactive Oxygen and Nitrogen Species," Toxicology, 276 (2), 85-94.

Romero-Avila, D. \& Roche, E. (1998). "The Keys of Oxidative Stress in Acquired Immune Deficiency Syndrome Apoptosis," Medical Hypothesys, 51, 169-173.

Sahnoun, Z., Jamoussi, K. \& Zeghal, K. M. (1997). "Free Radicals and Antioxidants: Human Physiology, Pathology and Therapeutics Aspects," Therapie, 52, 251270.

Sloand, E. M., Young, N. S., Kumar, P., Weichold, F. F., Sato, T. \& Maciejewski, J. P. (1997). "Role of Fas Ligand and Receptor in the Mechanism of T-Cell Depletion in Acquired Immunodeficiency Syndrome: Effect on CD4+ Lymphocyte Depletion and Human Immunodeficiency Virus Replication," Blood, 89, 1357-1363.

Sonnerborg, A., Carlin, G., Akerlund, B. \& Jarstrand, C. (1988). "Increased Production of Malondialdehyde in Patients with HIV Infection," Scandinavian Journal of Infectious Diseases, 20, 287-290.

Stephensen, C. B., Marquis, G. S., Douglas, S. D., Kruzich, L. A. \& Wilson, C. M. (2007). "Glutathione, Glutathione Peroxidase, and Selenium Status in HIV-Positive and HIVNegative Adolescents and Young Adults," The American Journal of Clinical Nutrition, 5(1), 173.

Stephens, W. E. (2004). "Oxidative Stress in Viral Hepatitis and AIDS," Experimental Molecular Pathology, 77, 121-132.

Sundaram, M., Saghayam, S., Priya, B., Venkatesh, K. K. \& Balakrishnan, P. (2008). "Changes in Antioxidant Profile among HIVInfected Individuals on Generic Highly Active Antiretroviral Therapy in Southern India," International Journal of Infection Disease, 12, 61- 66.

Tang, A. M., Lanzillotti, J., Hendricks, K., Gerrior, J., Ghosh, M., Woods, M. \& Wanke,
M. (2005). "Micronutrients: Current Issues for HIV Care Providers," AIDS, 19, 847-861.

Tchkonia, T., Morbeck, D. E., Von Zglinicki, T., Van Deursen, J., Lustgarten, J., Scrable, H., Khosla, S., Jensen, M. D. \& Kirkland, J. L. (2010). "Fat Tissue, Aging, and Cellular Senescence," Aging Cell, 9, 667-684.

Trinei, M., Giorgio, M., Cicalese, A., Barozzi, S., Ventura, A., Migliaccio, E., Milia, E., Padura, I. M., Raker, V. A. \& Maccarana, M. (2002). "A P53-P66 Shc Signalling Pathway Controls Intracellular Redox Status, Levels of Oxidation-Damaged DNA and Oxidative Stress-Induced Apoptosis," Oncogene, 21(24), 3872-3878.

UNAIDS. AIDS Epidemic Update 2011. Available at: http://www.unaids.org

Ushio-Fukai, M. (2009). "Compartmentalization of Redox Signaling through NADPH Oxidase-Derived ROS," Antioxidant Redox Signal, 11, 1289-1299.

Valko, M., Leibfritz, D., Moncol, J., Cronin, M. T., Mazur, M. \& Telser, J. (2007). "Free Radicals and Antioxidants in Normal Physiological Functions and Human Disease," International Journal of Biochemical and Cell Biology, 39, 44 -84.

Vassimon, H. S., Deminice, R., Machado, A. A., Monteiro, J. P. \& Jordão, A. (2010). "The Association of Lipodystrophy and Oxidative Stress Biomarkers in HIV-Infected Men," Current HIV Research, 8(5), 364 -369.

Wanchu, A., Rana, S. V., Pallikkuth, S. \& Sachdeva, R. K. (2009). "Oxidative Stress in HIV-Infected Individuals: A Cross-Sectional Study," AIDS Research and Human Retroviruses, 25, 1307-1311.

WHO: Global Summary of AIDS Epidemic, 2011.

http://www.who.int/hiv/data/2011_global_ summary.png.

Yousefi, S., Hadadi, A., Ostovar, A., Noor, B. E., Rasoolinejad, M., Abdolbaghi, M. H. \& Khalili, H. (2011). "Protocol of Determining the Effect of Selenium Supplementation on CD4 + T Lymphocyte Count in HIV/AIDS Patients: A Randomized Double Blind Placebo Controlled Trial," Advances in Infectious Diseases, 1, 20-26. 